F. D. Tollenan. Possibilities and limitations of the use of antioxidants in edible oils and fats. IXe Cong. Intern. Ind. Agric., Rome, 1952, T. P. 9.

F. D. Tollenaar et H. J. Vos. Use of butylated hydroxytoluene as an antioxidant in foods. Fette, Seifen Anstrichmitt, 1956, 58 (2), 112, cité par D. S. A., 1959, 21 (6), 272.

P. H. Tracy, W. A. Hoskisson et J. M. Trimble. Wheat germ oil as an antioxidant in dairy products. J. Dairy Sci., 1944, 27, 311.

G. M. Trout et B. R. Weinstein. The solar activated flavor of homogenized milk. XIII th Intern. Dairy Cong. The Hague, 1953, 3, 1061.

G. A. Ulex et E. .P. Kröger. Uber die Wirksamkeit der Nordihydroguajaretsäure als Antioxidant für Fette. Dtsch. Lebensmitt. Rdsch., 1950, 46 (11), 256, eité par D. S. A., 1951, 13 (4), 532.

F. E. Van Haeften et J. W. Pette. Cold storage experiments on butter. XIIIth Intern. Dairy Cong. The Hague, 1953, 2, 541.

R. WaIte. The keeping quality of milk powders. J. Dairy Res., 1941, 12, 178.

J. C. D. White, J. A. B. Smith et C. H. Lea. The effect of a high preheating temperature with and without ethyl gallate on the storage life of whole milk powder. J. Dairy Res., 1947, 15, 127.

P. A. Wright et G. R. Greenbank. The effect of the ascorbic acid content of fluid milk upon the keeping quality of its dried product. J. Dairy Sci., 1949, 32, 644.

\title{
CONTRIBUTION A L'ÉTUDE DE L'ACTION DE LA CHALEUR SUR LES CASÉINES (Fin)
}

\author{
par
}

Mme M. SWIRSKI, R. ALLOUF et H. CHEFTEL

Laboratoire de Recherches des Etablissements J.-J. Carnaud et Forges de Basse-Indre

\section{II. - Influence du chauffage sur l'hydrolyse enzymatique}

\section{A. - Hydrolyse trypsique}

\section{Mise en liberté d'azote aminé.}

Les dosages ont été effectués après $3,24,48,72$ et 100 heures d'hydrolyse. La figure 4 résume nos résultats : en l'absence de chauffage préalable, l' $\alpha$-caséine est hydrolysée bien plus rapidement que la caséine totale; le chauffage ralentit considérablement l'hydrolyse tant de l'une que de l'autre ; la $\beta$-caséine, au contraire, est relativement inerte vis-à-vis de la trypsine, ainsi que CHRIstensen [6] et 
TABLEAU II

TENEUR EN GOMPOSÉS PHOSPHORÉS NON DIALYSABLES DE SOLUTIONS DE GASÉINES NON GHAUFFÉE ET PREALABLEMENT GHAUFFÉS

\begin{tabular}{|c|c|c|c|}
\hline ' & $\begin{array}{c}\text { Avant } \\
\text { chauffage } \\
\text { P p. } 100 \\
\text { de protéine }\end{array}$ & $\begin{array}{c}\text { Après } \\
\text { chauffage } \\
\text { P p. } 100 \\
\text { de protéine }\end{array}$ & $\begin{array}{l}\text { Composés } \\
\text { phosphorés } \\
\text { libérés p. } 100 \\
\text { de P total }\end{array}$ \\
\hline Caséine totale sol. à 0,48 p. $100 \ldots$ & $0,89(1)$ & 0,50 & 44,0 \\
\hline Caséine totale sol. à 1,95 p. $100 \ldots$ & 0,87 & 0,59 & 32,2 \\
\hline$\alpha$-Caséine sol. à 0,52 p. $100 \ldots \ldots$ & $0,97(2)$ & 0,48 & 50,3 \\
\hline$\alpha$-Caséine sol. à 2,08 p. $100 \ldots \ldots$ & 0,96 & 0,53 & 44,6 \\
\hline$\beta$-Caséine sol. à 0,59 p. $100 \ldots \ldots$ & $0,63(2)$ & 0,58 & 7,9 \\
\hline
\end{tabular}

(1) Divers auteurs [28 bis] indiquent pour la teneur en phosphore de la caséine totale des valeurs comprises entre 0,71 et 0,87 p. 100 de protéine.

(2) Gordon et coll. [15 bis] ont trouvé, pour la teneur en phosphore des caséines $\alpha$ et $\beta$, respectivement 0,98 et 0,61 p. 100 .

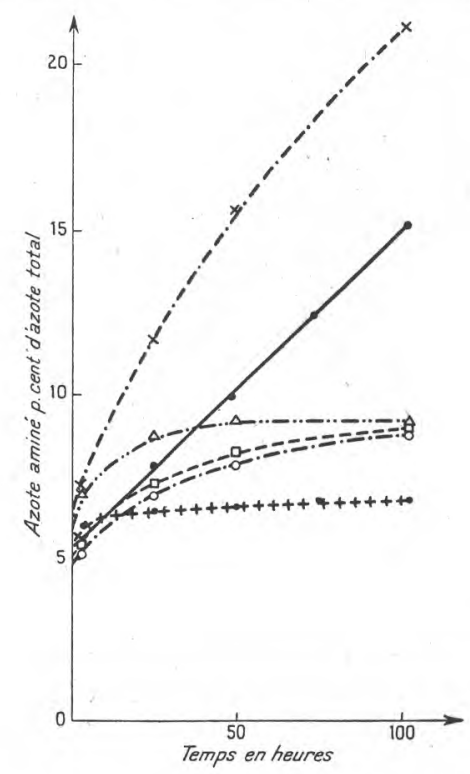

Figure 4. - Hydrolyse trypsique des caséines non chauffées et préalablement chauffées.

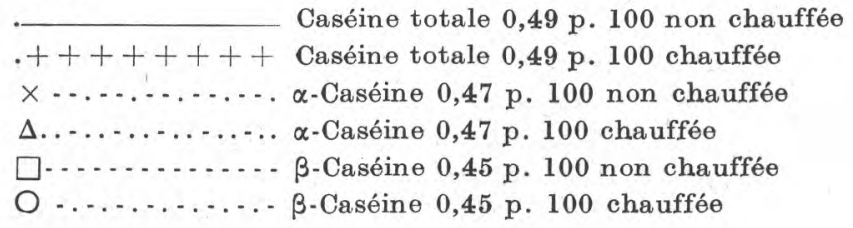


Lembke [25] l'avaient constaté après 2 heures seulement de protéolyse et ceci uniquement par des mesures de viscosité et par fractionnement électrophorétique; le chauffage ne modifie pas l'allure des courbes d'hydrolyse trypsique de la $\beta$-caséine.

L'allure de ces courbes nous a conduits à étudier l'hydrolyse trypsique de mélange d' $\alpha$ - et de $\beta$-caséine ; nous avons constaté que la présence de $\beta$-caséine ralentit plus ou moins l'hydrolyse de l' $\alpha$ -

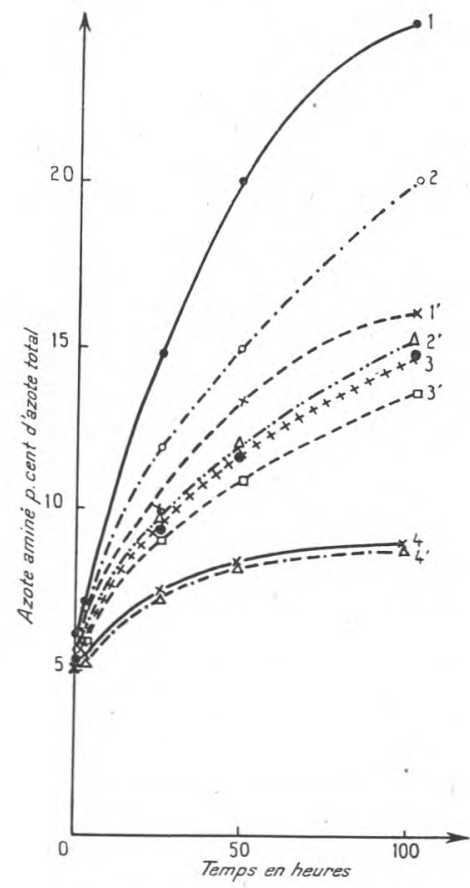

Figure 5. - Hydrolyse trypsique de mélanges d' $\alpha$-caséine et $\beta$-caséine non chauffés et préalablement chauffés.

$1 . \alpha$-Caséine à 1,8 p. 100 non chauffée

1 ' $\times \ldots . . . . . . . . .-$-Caséine à 1,8 p. 100 chauffée

$2 \bigcirc \ldots \ldots \ldots \ldots$-Caséine à $1,8 \mathrm{p} \cdot 100+\beta$-caséine à $0,45 \mathrm{p} .100$, non chauffées

2, $\Delta \ldots \ldots \ldots \ldots \ldots \ldots$-Caséine à 1,8 p. $100+\beta$-caséine à 0,45 p. 100 chauffées

$3 \cdot t+t+++++\alpha$-Caséine à 1,8 p. $100+\beta$-caséine à 0,9 p. 100 , non chauffées

$3^{\prime} \square \ldots \ldots \ldots \ldots . . . . .-$-Caséine à 1,8 p. $100+\beta$-caséine à 0,9 p. 100 chauffées

$4 \times-\beta$-Caséine à 0,9 p. 100 non chauffée

4' $\Delta \ldots \ldots \ldots \ldots . . . .$. -Caséine à 0,9 p. 100 chauffée 
caséine suivant les proportions respectives de ces deux fractions (fig. 5) et diminue l'action du chauffage sur cette hydrolyse. Cette influence de la $\beta$-caséine sur le comportement de l' $\alpha$-caséine nous paraît devoir être rapprochée de l'observation de McMeckin [28] selon laquelle la présence de $\beta$-caséine diminue la mobilité électrophorétique de l' $\alpha$-caséine. Cet auteur en conclut que ces deux protéines réagissent l'une avec l'autre.

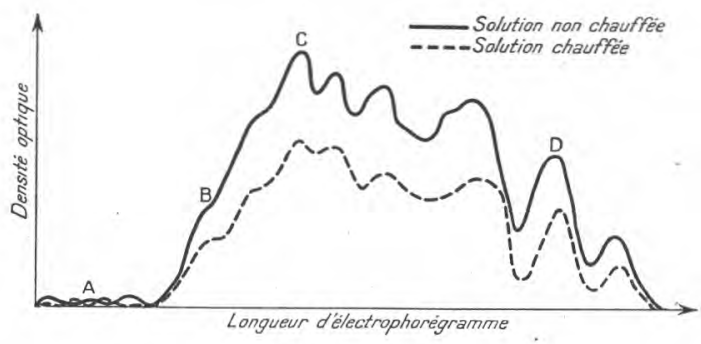

Figure 6. - Electrophorégrammes de solutions de caséine totale, non chauffée et préalablement chauffée après protéolyse trypsique de 3 heures, enregistrés par spectrophotométrie.
$A=$ acide aspartique
$\mathrm{C}=$ acides aminés neutres
$\mathrm{B}=$ acide glutamique
$\mathrm{D}=$ acides aminés basiques

2. Mise en liberté de composés azotés dialysables.

Etudiée uniquement sur la caséine totale à titre de vérification des expériences précédentes, elle les confirme de façon satisfaisante.

L'azote initialement présent dans la solution, et qui est en totalité non dialysable, passe plus rapidement sous la forme dialysable que sous la forme aminée; ceci est la conséquence de la libération rapide de peptides dialysables, et doit être rapproché des expériences dont nous rendons compte plus loin à propos de la libération des composés phosphorés dialysables.

\section{Recherche des acides aminés mis en liberté.}

La chromatographie sur papier confirme l'effet inhibiteur du chauffage sur l'hydrolyse trypsique de la caséine totale, et montre que le ralentissement observé dans la libération de l'azote aminé traduit, pour une part tout au moins, un retard dans la libération des divers acides aminés, et plus particulièrement de certains d'entre eux. 


\section{Fractionnement des acides aminés par électrophorèse.}

Le fractionnement en 4 groupes (ac. aspartique, ac. glutamique, ac. aminés neutres, ac. aminés basiques) selon BoulaNGER et Biserte [5], après 3,24 et 100 heures d'hydrolyse trypsique, révèle d'après les courbes densitométriques des électrophorégrammes des différences qualitatives et quantitatives entre caséine non chauffée et caséine chauffée (fig. 6, 7 et 8). Avec la caséine chauffée on observe un retard dans l'apparition de l'acide aspartique ainsi qu'une proportion notablement plus grande de peptides qui augmente au fur et à mesure que la protéolyse progresse.

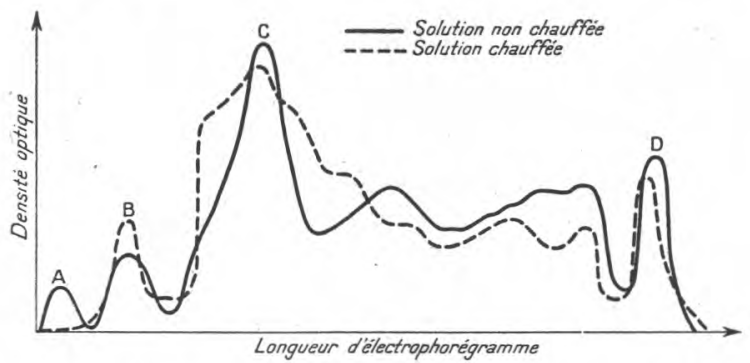

Figure 7. - Electrophorégrammes de solutions de caséine totale, non chauffée et préalablement chauffée, après protéolyse trypsique de 24 heures enregistrés par spectrophotométrie.
$\mathrm{A}=$ acide aspartique
$\mathrm{C}=$ acides aminés neutres
$\mathrm{B}=$ acide glutamique
$\mathrm{D}=$ acides aminés basiques

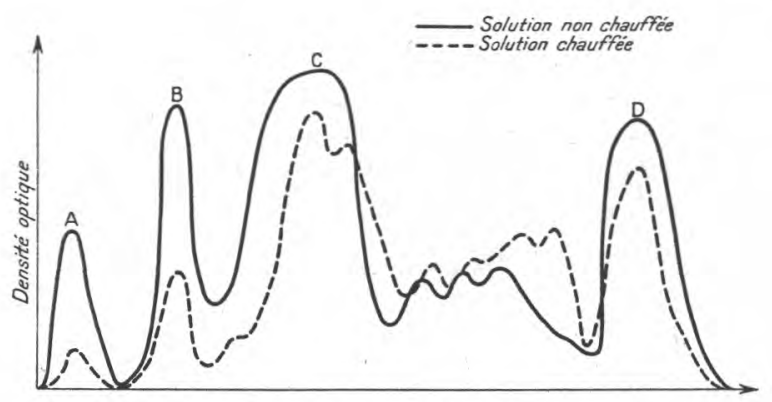

Longueur d'électrophorégramme

Figure 8. - Electrophorégrammes de solutions de caséine totale, non chauffée et préalablement chauffée, après protéolyse trypsique de 100 heures, enregistrés par spectrophotométrie.

\section{Longueur d'électrophorégramme}
$\mathrm{A}=$ acide aspartique
C = acides aminés neutres
$\mathrm{B}=$ acide glutamique
$\mathrm{D}=$ acides aminés basiques 


\section{Dosage des acides aminés libres ou engagés dans des peptides.}

En raison des difficultés de séparation, dues à la présence de peptides, nous n'avons dosé que cinq des acides aminés libérés : acide aspartique, acide glutamique, histidine, lysine, arginine.

D'autre part, les acides aminés constituant la fraction non précipitable par l'acide perchlorique ont été dosés après hydrolyse acide. Les dosages ont été effectués après 3, 24 et 100 heures d'hydrolyse trypsique.

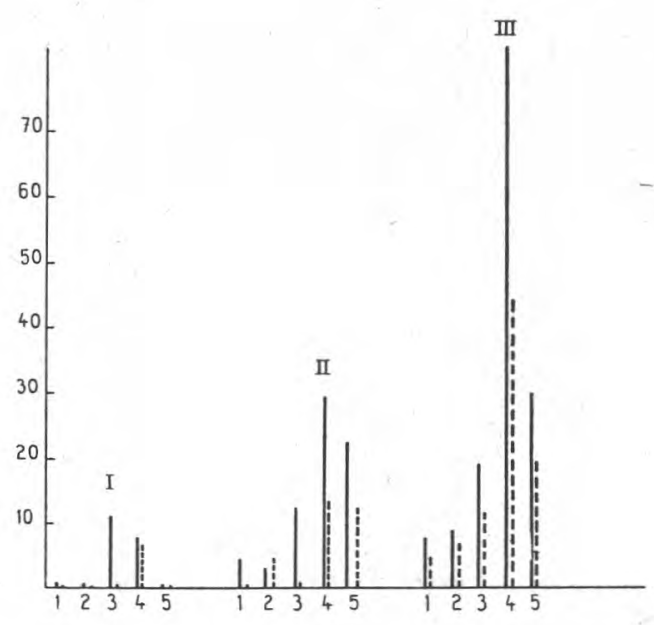

Figure 9. - Influence du chauffage sur la teneur en acides aminés à l'état libre après hydrolyse trypsique d'une solution de caséine à 2 p. 100.'

\begin{tabular}{|c|c|c|}
\hline \multicolumn{2}{|c|}{ Durée de protéolyse } & Acides aminés \\
\hline I & 3 heures & 1. Ac. aspartique \\
\hline II & 24 heures & 2. Ac. glutamique \\
\hline III & 100 heures & $\begin{array}{l}\text { 3. Histidine } \\
\text { 4. Lysine } \\
\text { 5. Arginine }\end{array}$ \\
\hline
\end{tabular}

Solution

Trait plein : non chauffée

Pointillé : chauffée

Les figures 9 et 10 et le Tableau III résument nos résultats : on remarque d'une façon générale que le chauffage préalable de la caséine retarde la libération des acides aminés, tout particulièrement des acides aminés basiques; seule la valine, la proline et la méthionine sont libérées à peu près à la même vitesse, que la caséine ait été chauffée ou non. 
6. Mise en liberté de composés phosphorés dialysables.

Nous avons étudié la libération des composés phosphorés sur la caséine totale et sur l' $\alpha$-et la $\beta$-caséine.
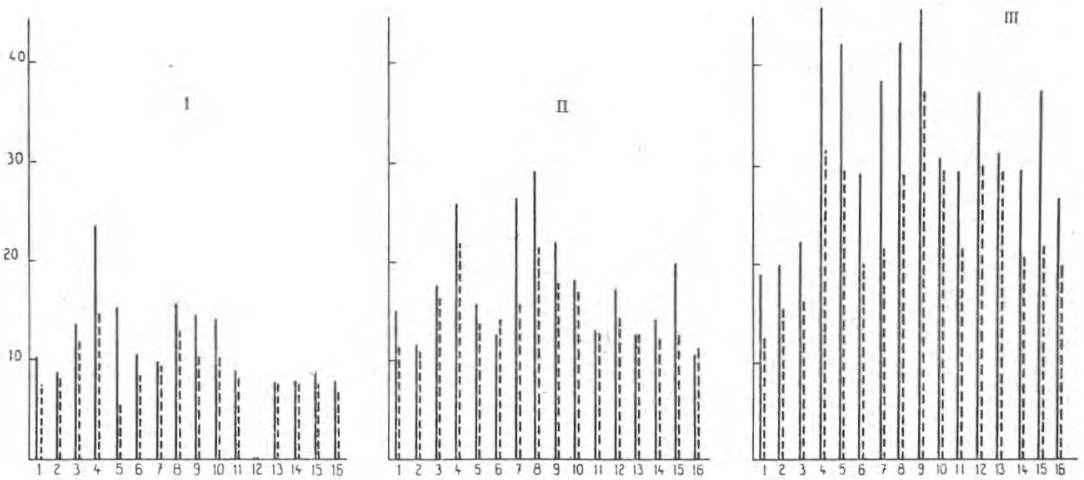

Figure 10. - Influence du chauffage sur la teneur en acides aminés après hydrolyse trypsique d'une solution de caséine à 2 p. 100 : acides aminés, libérés par hydrolyse acide, présents dans la fraction d'hydrolysat trypsique non précipitable par l'acide perchlorique, retenue sur Dowex 50 acide,

Durée de protéolyse

I 3 heures

II 24 heures

III 100 heures
1. Ac. aspartique

2. Ac. glutamique

3. Histidine

4. Lysine

5. Arginin $\theta$

6. Leucines

7. Glycin $\theta$

8. $\alpha$-alanin $\theta$ Solution

Trait plein : non chauffée

Pointillé : chauffée
9. Valine

10. Proline

11. Phénylalanine

12. Cystine

13. Méthionin $\theta$

14. Sérine

15. Thréonine

16. Tyrosine

Caséine totale et $\alpha$-caséine conduisent aux mêmes observations : une proportion importante du phosphore est libérée sous forme dialysable, dans des composés de nature organique, fait déjà signalé par Grabar [16], Damodarañ [9] et Posternak [43] à l'occasion de travaux portant sur la libération de phosphopeptides par hydrolyse trypsique; la proportion de phosphore libéré augmente plus rapidement pendant les premières 24 heures d'hydrolyse trypsique, et plus pour les solutions à 0,5 p. 100 que pour celles à 2 p. 100 de caséine; l'étude de l'influence du chauffage préalable de la caséine sur la libération des composés phosphorés conduit aux conclusions suivantes : 
TABLEAU III

INFLUENGE DU GHAUFFAGE SUR LA TENEUR EN AGIDES AMiNÉS APRÈS HYDROLYSE TRYPSIQUE D'UNE SOLUTION DE CASÉINE A 2 P. 100

\begin{tabular}{|c|c|c|c|c|c|c|c|c|c|c|c|c|c|c|c|c|c|c|c|c|c|c|c|c|}
\hline & \multicolumn{24}{|c|}{ Temps de protéolyse } \\
\hline & \multicolumn{8}{|c|}{3 heures } & \multicolumn{8}{|c|}{24 heures } & \multicolumn{8}{|c|}{100 heures } \\
\hline & \multicolumn{4}{|c|}{ Non chauffée } & \multicolumn{4}{|c|}{ Chauffée } & \multicolumn{4}{|c|}{ Non chauffée } & \multicolumn{4}{|c|}{ Chauffée } & \multicolumn{4}{|c|}{ Non chauffée } & \multicolumn{4}{|c|}{ Chauffée } \\
\hline & \multicolumn{2}{|r|}{ I } & \multicolumn{2}{|c|}{ II } & \multicolumn{2}{|r|}{ I } & \multicolumn{2}{|c|}{ II } & \multicolumn{2}{|c|}{ I } & \multicolumn{2}{|c|}{ II } & \multicolumn{2}{|c|}{ I } & \multicolumn{2}{|c|}{ II } & \multicolumn{2}{|r|}{ I } & \multicolumn{2}{|c|}{ II } & \multicolumn{2}{|c|}{ I } & \multicolumn{2}{|c|}{ II } \\
\hline & $a$ & $b$ & $a$ & $b$ & $a$ & $b$ & $a$ & $b$ & $a$ & $b$ & $a$ & $b$ & $a$ & $b$ & $a$ & $b$ & $a$ & $b$ & $a$ & $b$ & $a$ & $b$ & $a$ & $b$ \\
\hline 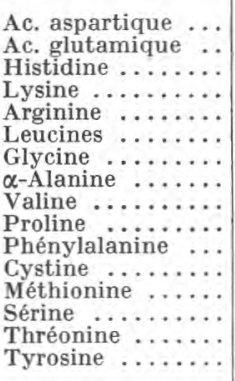 & $\begin{array}{r}<1,1 \\
<0,4 \\
5,6 \\
4,0 \\
<2,4\end{array}$ & $\begin{array}{r}0,07 \\
<0,08 \\
0,15 \\
0,27 \\
<0,07\end{array}$ & $\begin{array}{r}10,0 \\
9,6 \\
13,7 \\
23,7 \\
15,3 \\
10,7 \\
10,0 \\
15,8 \\
14,5 \\
14,1 \\
9,1 \\
<9,0 \\
7,4 \\
7,9 \\
9,4 \\
7,9\end{array}$ & $\begin{array}{c}0,66 \\
1,96 \\
0,37 \\
1,62 \\
0,44 \\
1,48 \\
0,20 \\
0,55 \\
1,03 \\
1,40 \\
0,45 \\
<0,005 \\
0,15 \\
0,56 \\
0,33 \\
0,41\end{array}$ & $\begin{array}{r}<1,1 \\
<0,4 \\
<5,6 \\
3,1 \\
<2,4\end{array}$ & $\begin{array}{r}<0,07 \\
<0,08 \\
<0,15 \\
0,21 \\
<0,07\end{array}$ & $\begin{array}{r}7,8 \\
8,3 \\
11,7 \\
14,6 \\
5,3 \\
8,5 \\
9,2 \\
12,4 \\
10,3 \\
10,2 \\
8,2 \\
<9,0 \\
7,9 \\
7,4 \\
7,6 \\
6,8\end{array}$ & $\begin{array}{c}0,52 \\
1,70 \\
0,32 \\
0,99 \\
0,15 \\
1,14 \\
0,18 \\
0,43 \\
0,74 \\
1,01 \\
<0,40 \\
<0,005 \\
0,16 \\
0,53 \\
0,26 \\
0,35\end{array}$ & $\begin{array}{r}2,3 \\
1,6 \\
6,2 \\
14,6 \\
10,7\end{array}$ & $\begin{array}{l}0,15 \\
0,33 \\
0,16 \\
0,99 \\
0,31\end{array}$ & \begin{tabular}{l|l}
14,6 & \\
11,5 & 2 \\
17,9 & 0 \\
25,9 & 1 \\
15,7 & \\
12,8 & 1 \\
26,3 & \\
28,3 & \\
21,6 & \\
18,1 & \\
13,2 & \\
16,9 & \\
12,7 & \\
14,1 & \\
19,5 & \\
10,8 &
\end{tabular} & $\begin{array}{l}0,96 \\
2,35 \\
0,49 \\
1,98 \\
0,46 \\
1,72 \\
0,57 \\
0,99 \\
1,53 \\
1,80 \\
0,65 \\
0,01 \\
0,27 \\
1,00 \\
0,68 \\
0,56\end{array}$ & $\begin{array}{r}<1,1 \\
2,0 \\
<5,6 \\
6,5 \\
5,8\end{array}$ & $\begin{array}{r}<0,07 \\
0,40 \\
<0,15 \\
0,44 \\
0,17\end{array}$ & $\begin{array}{l}11,4 \\
10,8 \\
16,0 \\
21,9 \\
13,5 \\
14,1 \\
15,3 \\
21,2 \\
17,8 \\
17,5 \\
12,6 \\
14,3 \\
12,5 \\
12,8 \\
12,8 \\
11,2\end{array}$ & $\begin{array}{l}0,75 \\
2,19 \\
0,43 \\
1,49 \\
0,39 \\
1,89 \\
0,30 \\
0,72 \\
1,26 \\
1,74 \\
0,62 \\
0,008 \\
0,26 \\
0,91 \\
0,45 \\
0,58\end{array}$ & $\begin{array}{r}3,7 \\
4,8 \\
9,8 \\
41,5 \\
14,8 \\
14,3 \\
9,2 \\
19,2 \\
14,8 \\
9,5 \\
7,2 \\
<9,0 \\
8,6 \\
9,5 \\
15,5 \\
12,4\end{array}$ & $\begin{array}{c}0,24 \\
0,97 \\
0,27 \\
2,83 \\
0,43 \\
1,92 \\
0,18 \\
0,67 \\
1,05 \\
0,94 \\
0,35 \\
<0,005 \\
0,18 \\
0,67 \\
0,54 \\
0,62\end{array}$ & $\begin{array}{l}18,4 \\
19,2 \\
22,0 \\
45,2 \\
42,1 \\
28,4 \\
38,4 \\
41,8 \\
43,2 \\
30,5 \\
29,4 \\
37,4 \\
30,6 \\
29,8 \\
37,4 \\
25,8\end{array}$ & $\begin{array}{l}1,20 \\
3,89 \\
0,59 \\
3,08 \\
1,23 \\
3,82 \\
0,77 \\
1,45 \\
3,07 \\
3,03 \\
1,44 \\
0,02 \\
0,64 \\
2,12 \\
1,32 \\
1,35\end{array}$ & $\begin{array}{r}1,8 \\
3,4 \\
5,8 \\
22,1 \\
9,5 \\
\\
\end{array}$ & $\begin{array}{c}0,12 \\
0,69 \\
0,16 \\
1,50 \\
0,28 \\
\end{array}$ & $\begin{array}{l}12,3 \\
15,1 \\
16,0 \\
31,3 \\
28,5 \\
19,6 \\
21,2 \\
29,5 \\
37,0 \\
28,4 \\
21,5 \\
28,5 \\
29,8 \\
20,5 \\
21,2 \\
19,7\end{array}$ & $\begin{array}{l}0,81 \\
3,06 \\
0,43 \\
2,13 \\
0,82 \\
2,64 \\
0,42 \\
1,04 \\
2,62 \\
2,82 \\
1,10 \\
0,02 \\
0,62 \\
1,46 \\
0,74 \\
1,03\end{array}$ \\
\hline
\end{tabular}

I = Acides aminés à l'état libre

présents dans la fraction d'hydrolysat trypsique non précipitable par l'acide perchlorique et retenue sur

$a=$ Proportion p. 100 rapportée à la quantité de l'acide aminé existant dans la caséine à l'origine.

$\begin{aligned} & a \\ & b\end{aligned}=$ P. 100 de caséine. 
TABLEAU IV

\begin{tabular}{|c|c|c|c|c|c|c|c|c|c|c|c|c|c|c|c|c|c|c|c|c|}
\hline & \multicolumn{8}{|c|}{ Caséine totale } & \multicolumn{8}{|c|}{$\alpha$-Caséine } & \multicolumn{4}{|c|}{$\beta$-Caséine } \\
\hline & \multicolumn{4}{|c|}{$[\mathrm{N}]=2,950 \mathrm{~g} / \mathrm{l}$} & \multicolumn{4}{|c|}{$[\mathrm{N}]=0,760 \mathrm{~g} / \mathrm{l}$} & \multicolumn{4}{|c|}{$[\mathrm{N}]=2,24 \mathrm{~g} / \mathrm{l}$} & \multicolumn{4}{|c|}{$[\mathrm{N}]=0,56 \mathrm{~g} / \mathrm{l}$} & \multicolumn{4}{|c|}{$[\mathrm{N}]=0,49 \mathrm{~g} / \mathrm{l}$} \\
\hline & \multicolumn{2}{|c|}{\begin{tabular}{|c|} 
Non chauffée \\
{$[\mathrm{P}]$ total : } \\
$166,7 \mathrm{mg} / \mathrm{l}$
\end{tabular}} & \multicolumn{2}{|c|}{$\begin{array}{l}\text { Chauffée } \\
\text { [P] total : } \\
108,0 \mathrm{mg} / 1\end{array}$} & \multicolumn{2}{|c|}{$\begin{array}{c}\text { Non chauffée } \\
{[\mathrm{P}] \text { total : }} \\
43,0 \mathrm{mg} / \mathrm{l}\end{array}$} & \multicolumn{2}{|c|}{$\begin{array}{l}\text { Chauffée } \\
\text { [P] total : } \\
23,9 \mathrm{mg} / 1\end{array}$} & \multicolumn{2}{|c|}{$\begin{array}{l}\text { Non chauffée } \\
{[\mathrm{P}] \text { total : }} \\
142,0 \mathrm{mg} / \mathrm{l}\end{array}$} & \multicolumn{2}{|c|}{$\begin{array}{l}\text { Chauffée } \\
\text { [P] total : } \\
76,5 \mathrm{mg} / \mathrm{l}\end{array}$} & \multicolumn{2}{|c|}{$\begin{array}{c}\text { Non chauffée } \\
{[\mathrm{P}] \text { total : }} \\
34,6 \mathrm{mg} / \mathrm{l}\end{array}$} & \multicolumn{2}{|c|}{$\begin{array}{l}\text { Chauffée } \\
{[\mathrm{P}] \text { total : }} \\
17,1 \mathrm{mg} / 1\end{array}$} & \multicolumn{2}{|c|}{$\begin{array}{c}\text { Non ehauffée } \\
{[\mathrm{P}] \text { total : }} \\
21,4 \mathrm{mg} / \mathrm{l}\end{array}$} & \multicolumn{2}{|c|}{$\begin{array}{l}\text { Chauffée } \\
{[P] \text { total : }} \\
16,8 \mathrm{mg} / \mathrm{l}\end{array}$} \\
\hline Temps heures & I & II & I & II & I & II & I & II & I & II & I & II & I & II & I & II & I & II & I & II \\
\hline$\ldots \ldots \ldots$ & 0 & 0 & 0 & 0 & 0 & 0 & 0 & 0 & 0 & 0 & 0 & 0 & 0 & 0 & 0 & 0 & 0 & 0 & 0 & 0 \\
\hline $3 \ldots \ldots \ldots$ & 20,0 & 11,9 & 13,8 & 12,7 & 7,4 & 17,2 & 7,6 & 31,8 & 11,2 & 7,8 & 9,3 & 12,1 & 7,4 & 21,4 & 3,1 & 18,1 & 4,5 & 21,0 & 3,1 & 18,4 \\
\hline $24 \ldots \ldots \ldots$ & 33,6 & 20,0 & 24,1 & 22,3 & 14,2 & 33,0 & 8,0 & 33,5 & 52,1 & 33,6 & 28,8 & 37,8 & 14,3 & 41,3 & 6,0 & 35 & 4,5 & 21,0 & 2,4 & 14,3 \\
\hline $100 \ldots \ldots \ldots$ & 75,2 & 45,1 & 55,8 & 51,6 & 23,6 & 54,9 & 11,2 & 46,9 & 62,9 & 44,3 & 37,9 & 49,5 & 18,2 & 52,5 & 8,2 & 47,9 & 4,5 & 21,0 & 3,0 & 17,8 \\
\hline
\end{tabular}

II $=$ Phosphore sous forme dialysable, p. 100 de phosphore total. 
10 La quantité de phosphore dialysable libéré par protéolyse est. moins grande dans le cas de la caséine chauffée ;

$2^{\circ}$ Si l'on rapporte le phosphore dialysable libéré par protéolyse à la teneur initiale en phosphore de la caséine mise en œuvre, on note au contraire que le chauffage a pour effet d'en augmenter la proportion. Cette apparente contradiction tient, ainsi que nous l'avons signalé plus haut, au fait que le chauffage per se libère déjà une fraction importante de phosphore sous la forme minérale, de telle sorte que l'action protéolytique s'exerce sur une caséine déjà appauvrie en phosphore du fait du simple chauffage.

La $\beta$-caséine d'autre part, cède peu de phosphore, et uniquement au début de l'hydrolyse trypsique ; son chauffage ne modifie pas son comportement. Ces résultats étaient prévisibles d'après les observations faites précédemment sur cette fraction de la caséine.

\section{B. - Hydrolyse pepsique}

Etudiée uniquement sur la caséine totale, par dosage de l'azote aminé et de l'azote dialysable mis en liberté, elle nous a permis de constater que le chauffage préalable n'entraîne pas de modification. Nous n'avons pas cru devoir procéder à une étude plus détaillée.

\section{GONGLUSIONS}

L'influence du traitement thermique sur certaines propriétés physiques et chimiques de la caséine totale, de l' $\alpha$-caséine, et de la $\beta$-caséine a été étudiée. Les modifications apportées à l'hydrolyse trypsique et pepsique de ces caséines par le chauffage prolongé en milieu tamponné $p H$ 7,2 ont également été analysées.

Les conclusions qui se dégagent peuvent être résumées ainsi :

\section{Propriétés physiques et composition.}

Le traitement thermique modifie la couleur, la solubilité en fonction du $p \mathrm{H}$, la teneur en phosphore, de la caséine totale et de l' $\alpha$-caséine; ces caractéristiques demeurent inchangées dans le cas de la $\beta$-caséine.

La constante de sédimentation de l' $\alpha$-caséine est augmentée par le chauffage, celle de la $\beta$-caséine ne varie pas.

L'absorption dans l'ultra-violet entre 2600 et $2800 \AA$ augmente sous l'effet du chauffage dans le cas de la $\beta$-caséine, elle n'est pas modifiée dans le cas de la caséine totale et de l' $\alpha$-caséine. 
Ia composition en acides aminés de la caséine totale n'est pas modifiée par le chauffage.

\section{Hydrolyse trypsique.}

L'étude de l'hydrolyse trypsique a porté sur la caséine totale, l' $\alpha$-et la $\beta$-caséine.

Dans le cas de la caséine totale, l'activité enzymatique a été suivie par des dosages d'acides aminés et de peptides libérés; pour l' $\alpha$ - et la $\beta$-caséine, seul l'azote aminé total a été déterminé.

Le chauffage ralentit la mise en liberté des acides aminés acides et basiques. L'influence du traitement thermique est surtout notable pour l'histidine; après trois heures d'hydrolyse des traces seulement en sont détachées de la caséine chauffée, alors que plus de 5 p. 100 de la quantité initiale d'histidine sont libérés d'une caséine non chauffée. La lysine se comporte de façon analogue, 22 p. 100 de cet acide aminé sont libérés après 100 heures d'hydrolyse après chauffage, contre 41 p. 100 sans chauffage.

Le ralentissement de l'hydrolyse enzymatique de la caséine totale par le chauffage est confirmé par les dosages :

1. d'acides aminés libres dibasiques et diacides;

2. Des acides aminés obtenus après hydrolyse acide de la fraction non précipitable par l'acide perchlorique.

Pour la plupart des 16 acides aminés dosés, les nombres sont d'une façon globale de 20 à 40 p. 100 inférieurs après chauffage. Cependant l'effet du traitement thermique n'est pas uniforme. Ainsi la vitesse de libération de la valine, de la proline et de la méthionine demeure pratiquement inchangée, que la solution de caséine totale ait été ou non préalablement chauffée.

L'hydrolyse enzymatique de l' $\alpha$-caséine est notablement ralentie après chauffage. Il n'en est pas de même avec la $\beta$-caséine. Il en ressort que l'effet inhibiteur du chauffage sur l'hydrolyse enzymatique de la caséine totale paraît s'exercer surtout sur la fraction $\alpha$-caséine. L'addition de $\beta$-caséine à une solution d' $\alpha$-caséine a pour effet d'inhiber l'attaque trypsique de cette dernière, et de réduire, plus ou moins suivant la quantité de $\beta$-caséine ajoutée, l'influence du chauffage sur l'activité enzymatique.

Le simple chauffage réduit considérablement la teneur en phosphore de l'a-caséine, et influence relativement peu celle de la $\beta$-caséine. La quantité absolue de phosphore organique libéré par hydrolyse trypsique après chauffage diminue dans le cas de l' $\alpha$ caséine, et à un degré moindre avec la $\beta$-caséine. 


\section{Hydrolyse pepsique.}

Le chauffage ne modifie pas la libération de l'azote aminé ou de l'azote dialysable de la caséine totale soumise à une hydrolyse pepsique. Une analyse plus détaillée nous paraissait de ce fait inutile.

\section{RÉSUMÉ}

L'influence du chauffage sur certaines propriétés de la caséine totale, de l' $\alpha$-caséine et de la $\beta$-caséine, en solution tampon à $p H$ 7,2 a été étudiée.

Caséine totale et $\alpha$-caséine: couleur, solubilité en fonction du $p \mathrm{H}$, constante de sédimentation, teneur en phosphore, allure de l'hydrolyse trypsique, vitesse de libération des acides aminés (étudiée chimiquement sur la caséine totale) sont modifiées; l'absorption dans l'U. V. demeure inchangée.

ß-caséine: l'absorption dans l'U. V. augmente, les autres propriétés étudiées ne sont pas modifiées.

La $\beta$-caséine ajoutée à l' $\alpha$-caséine assure dans certaines conditions une protection de cette dernière contre l'action du chauffage.

L'hydrolyse pepsique de la caséine totale ne paraît pas modifiée.

\section{SUMMARY}

The effect of heating upon certain properties of whole casein, $\alpha$-casein and $\beta$-casein, in solution buffered at $p \mathrm{H} \mathrm{7,2,} \mathrm{has} \mathrm{been}$ investigated.

Whole casein and $\alpha$-casein : Colour, solubility as a function of $p \mathrm{H}$, sedimentation constant, phosphorus content, trend of the hydrolysis by trypsin, rate of liberation of amino-acids (studied chemically on whole casein) undergo modifications; U. V. absorption remains unaltered.

$\beta$-casein: U. V. absorption is increased, none of the other properties studied is modified.

$\beta$-casein, added to $\alpha$-casein, insures under certain conditions a protection of the latter against the effect of heating.

Pepsic hydrolysis of whole casein does not appear modified.

\section{BIBLIOGRAPHIE}

[1] S. AdAchi. Nature, 1956, 177, 936.

[2] R. Allouf. Thèse de doctorat, Paris, 1953.

[3] G. Bigerte, T. Plaquet-Schoonaert, P. Boulanger et P. Paysant. J. Chromatography, $1960,3,25$.

[4] R. J. BLock, D. B. Jones et G. E. F. Gersdonf. J. Biol, Chem., 1934, 105,667 . 
[5] P. Boulanger et G. Biserte. Bull. Soc. Chim. Biol., 1951, 33, 1930.

[6] L. R. Christensen. Arch. Biochem. and Biophys., 1954, 53, 128.

[7] W. Von DaM. Zeitschr, physiol. Chem., 1908, 58, 295.

[8] W. Von Dam. Zeitschr. physiol. Chem., 1912, 79, 247.

[9] M. Damodaran et B. V. Romochondran. Biochem. J., 1941, 35, 122.

[10] N. R. Eldied et G. Rodney. J. Biol. Chem., 1946, 162, 261.

[11] C. A. ElvenJ'em et A. G. Harper. J. Am. Med. Soc., 1955, $158,655$.

[12] C. A. Elvehлem. Federation Proc., 1956, 15, 965.

[13] L. Fowden. Biochem. J. 1952, 50, 355.

[14] R. Geiger. J. Nutr., 1947, 34, 97.

[15] E. GEIGER. Science, 1950, 111, 594.

[15 bis] W. G. Gordon, W. F. Semmet, R. S. Cable et M. Morris. J. Am. Chem. Soc., 1949, 71, 3293.

[16] P. Grabar. C. R. Soc. Biol., 1933, 114, 13.

[17] E. O. Greates, et A. F. Morgan. Proc. Soc. Exp. Biol. Anal. Med. $1934,31,506$.

[18] E. O. Greaves, A. F. Morgan et H. K. Loveen. J. Nutr., 1938, 16 115.

[19] G. R. Howat et N. C. Wright. Biochem. J., 1934, 28, 1336.

[20] G. R. Howat et N. C. Wright. Biochem. J. 1936, 30, 1413.

[21] Y. Inouz et K. Onodera. J. Ag. Chem. Soc. Japan, 1948, 22, 5, d'après C. A., $1951,9480 a$.

[22] M. A. Jermyn et F. A. Isherwood. Biochem. J., 1949, 44, 402.

[23] A. L. Krrk. Anal. Chem., 1950, 22, 355.

[24] J. K.jeldahl, Z. Anal. Chem., 1883, 22, 366.

[25] A. Lember et W. Kaufmann. Kieler Milchwirtsch. Forschungsber., 1953, 5, 173.

[26] Linderstrom-Lang. Cold Spring Harbor Symp. Quant. Biol., 1949, 64, 85 .

[27] E. V. Mçollum et M. J. Davis. J. Biol. Chem., 1917, 23, 247.

[28] T. L. McMecrin et B. D. Polis. Advances in Prot. Chem., 1949, 5, 204.

$[28$ bis $]$ T. L. McMeckin. - "The proteins", H. Neurath et K. Bailey. Acad. Press., New York, 1954, II A, chap. 16.

[29] M. Maki et H. Mesamune. J. exp. Med., 1952, 56, 245.

[30] F. W. MALPREss et A. B. Morrisson. Nature, 1949, 164, 963.

[31] A. Marletta. Biochem. Terap. Sper., 1929, 16, 297, d'après C. A., 1930,5804 .

[32] O. Meliander. Biochem. $Z$., 1939, 300, 240.

[33] D. MetnioK, B. L. Oser et S. Weiss. Ścience, 1946, 103, 326.

[34] D. Melnick et B. L. Oser. Food Technol., 1949, 3, 57.

[35] A. F. Morgan et V. A. Petro. J. Home. Econ., 1930, 22, 857.

[36] M. Pader, D. Melnick et B. L. Oser. J. Biol. Chem., 1948, 172, 763.

[37] L. S. Palmer. Proc. Soc. exp. Biol., 1921, $19,137$.

[38] S. Pappalardo. Bull. Soc. Ital. Biol. Spec., 1930, 5, 24, d'après C. A., $1930,4310$.

[39] J. K. Parnas et R.WAgner. Biochem. Z., 1921, 125, 253.

[40] S. M. Patridge. Biochem. J., 1948, 42, 248.

[41] K. O. Pedersen. Biochem. J., 1936, 30, 948. 
[42] Ch. Porcher. Le Lait, 1930, 10, 667.

[43] T. Posternak et H. Pollaczer. Helv. Chim. Acta, 1941, 24, 1190.

[44] M. Potterat et H. Eschman. Trav. Chim. Alim. et Hyg., 1954, 45, 312.

[45] F. W. Putnam, "The proteins", H. Neurath et K. Bailey. Acad.

Press, New York, 1953, I B, chap. 9.

[46] H. W. Schultz, W. H. Segegrs et H. A. Mattili. Proc. Soc.exp. Biol., $1935,32,1026$.

[47] R. C. WArner. J. Am. Chem. Soc., 1944, 66, 1725.

[48] A. J. Worwod et F. V. LingGood. Nature, 1949, 163, 218.

[49] M. M. Yenson. Bull. Fac. Med. Istambul, 1946, 9, 166, d'après C. A., $1947,1046 \mathrm{~h}$.

[50] Ch. Zinzadze. Ind. Eng. Chem. Anal. Ed., 1935, 7, 227.

\title{
SUPPLÉMENT TECHNIQUE
}

\section{L'EMPLOI DE L'ACIDE LACTIQUE DANS LA FABRICATION DE LA CASÉINE}

\author{
par \\ G. GÉNIN \\ Ingénieur E.P.C.I.
}

Depuis quelques années, l'industrie mondiale de la caséine a subi certaines modifications sous l'influence de facteurs économiques et techniques. Tout d'abord, les difficultés d'écoulement de la poudre de lait écrémé ont incité certains fabricants à convertir leur installation de fabrication de poudre en installation de fabrication de caséine, afin de trouver un écoulement rémunérateur au lait écrémé constituant un sous-produit de certaines de leurs fabrications. D'autre part, la consommation de la caséine ayant sensiblement évolué, certaines utilisations, comme la fabrication de la fibre de caséine, ont pratiquement disparu; par contre les emplois de la caséine comme matière de couchage dans l'industrie du papier se sont développés et enfin surtout la caséine est devenue une matière première pour certaines industries alimentaires, particulièrement sous la forme de son sel de sodium, ce qui a obligé les fabricants de caséine à livrer un produit plus pur que la caséine destinée à des applications techniques. Enfin, l'augmentation continuelle des dépenses de main-d'œurre a conduit certains producteurs à adopter un procédé de fabrication continu de la caséine, prenant 